\title{
Safety, Tolerability, and Pharmacokinetics of E3030, a Novel Peroxisome Proliferator-Activated Receptor $\alpha / \gamma$ Dual Agonist, in Healthy Japanese Male Subjects
}

\author{
Yutaka Takeuchi $^{1^{*}}$, Yuki Nishioka ${ }^{2}$, Yasumi Kitahara ${ }^{1}$, Setsuo Hasegawa ${ }^{3,4}$, Akihiro Ohnishi ${ }^{5}$ \\ ${ }^{1}$ Clinical Development, Japan/Asia Clinical Research PCU, Eisai Co., Ltd., Tokyo, Japan; ${ }^{2}$ Japan Regulatory Affairs, Global Regulatory \\ CFU, Eisai Co., Ltd., Tokyo, Japan; ${ }^{3}$ Sekino Clinical Pharmacology Clinic, Tokyo, Japan; ${ }^{4}$ Present address: Pharmaspur, Inc., Tokyo, \\ Japan; ${ }^{5}$ Department of Laboratory Medicine, Daisan Hospital, Jikei University School of Medicine, Tokyo, Japan. \\ Email: ${ }^{*}$ 2-takeuchi@hhc.eisai.co.jp
}

Received November $28^{\text {th }}, 2013$; revised December $29^{\text {th }}, 2013$; accepted January $14^{\text {th }}, 2014$

Copyright (C 2014 Yutaka Takeuchi et al. This is an open access article distributed under the Creative Commons Attribution License, which permits unrestricted use, distribution, and reproduction in any medium, provided the original work is properly cited. In accordance of the Creative Commons Attribution License all Copyrights (c) 2014 are reserved for SCIRP and the owner of the intellectual property Yutaka Takeuchi et al. All Copyright (C) 2014 are guarded by law and by SCIRP as a guardian.

\section{ABSTRACT}

Objective: The objectives of the present study were to evaluate the safety of single oral dose E3030 in healthy Japanese male subjects, and to evaluate pharmacokinetics after single oral dose E3030 and food effect on pharmacokinetic profiles. Methods: This study was conducted in a randomized, double-blind, placebo-controlled, ascending single-dose study in 56 healthy Japanese male subjects. Subjects were orally administered E3030 (0.5 $40 \mathrm{mg}$ ) or placebo. Results: Six of 42 (14\%) subjects' administered E3030 experienced adverse events; however, all adverse events were mild and transient, and there was no dose-dependent increase in any adverse event. Plasma samples were collected over 96 hours after dosing. After administration in the fasted state, $\mathrm{C}_{\max }$ of E3030 was achieved between 1.00 and 1.75 hours, indicating rapid absorption. Both $C_{\max }$ and AUC were dose-proportional in the range of $0.5 \mathrm{to} 40 \mathrm{mg}$. The average range of elimination half-life was $18.4-23.8 \mathrm{hr}$. CL/F and $\mathrm{V}_{\mathrm{z}} / \mathrm{F}$ also remained nearly constant regardless of dose levels. In addition, food effect was exploratorily evaluated in five subjects of administered E3030 (10 mg) in both fasted and fed states. The fed/fasted ratios for the geometric mean of the $\mathrm{C}_{\max }$ and AUC were 0.803 and 0.913 , respectively. Conclusion: E3030 was safe and well tolerated at single doses up to $40 \mathrm{mg}$. The pharmacokinetic profile showed good linearity, and food effect on pharmacokinetics of E3030 was not significant.

\section{KEYWORDS}

PPAR Agonist; Phase 1; Pharmacokinetics; Healthy Subjects

\section{Introduction}

Type 2 diabetes mellitus is frequently associated with abnormal lipid metabolism. Both hyperglycemia and dyslipidemia are known as pathogenic factors associated with atherosclerosis. Conventional pharmacotherapies for type 2 diabetes show little lipid-improving effect, although hypoglycemic effects are shown. Peroxisome proliferator-activated receptor (PPAR) $\alpha / \gamma$ dual agonists are considered highly attractive, because they can be used to treat both hyperglycemia and dyslipidemia. Pioglitazone, a PPAR $\gamma$ agonist, reduced the composite of "Corresponding author. all-cause mortality, non-fatal myocardial infarction and stroke in patients with type 2 diabetes having a high-risk of macrovascular events in the PRO active study [1]. Fenofibrate, a PPAR $\alpha$ agonist, did not significantly reduce the risk of the primary outcome of coronary events, but did reduce total cardiovascular events, mainly in fewer non-fatal myocardial infarctions and revascularizations in the FIELD study [2]. Although the ACCORD study reported no overall benefit for fenofibrate in combination with simvastatin [3], a meta-analysis suggested that fibrates can reduce the risk of major cardiovascular events predominantly by prevention of coronary events [4]. 
Therefore, PPAR $\alpha / \gamma$ dual agonists are expected theoretically to be more effective than either pioglitazone or fibrates alone in the prevention of cardiovascular events in diabetic patients. Until now, no PPAR $\alpha / \gamma$ dual agonists have been successfully launched into the market.

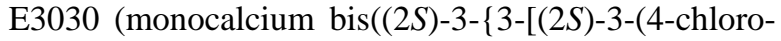
2-cyanophenoxy)-2-fluoropropoxy]phenyl $\}$-2-isopropoxypropionate) trihydrate) (Figure 1) is a novel PPAR $\alpha / \gamma$ dual agonist synthesized by Eisai Co., Ltd. [5]. E3030 activates both human $\operatorname{PPAR} \alpha$ and $\operatorname{PPAR} \gamma$ with $\mathrm{EC}_{50}$ values of 65 and $34 \mathrm{nmol} / \mathrm{L}$, respectively, in cell-based reporter gene assay. In addition to improvement of hyperglycemia and hyperinsulinemia in $d b / d b$ mice, E3030 remarkably reduces plasma triglyceride (TG) and nonhigh-density lipoprotein (non-HDL) cholesterol levels in beagle dogs in a manner similar to fenofibrate. Therefore, E3030 is expected to become a novel anti-diabetic drug that can control glucose and lipid levels as a single drug for patients with diabetes mellitus. Non-clinical studies using rat, dog, and human liver microsomes and recombinant human UDP-glucuronyltransferase isozymes suggested that the major metabolic pathway of E3030 was glucuronidation at carboxylic group. After a single oral administration of radiolabeled E3030 to rats and dogs, approximately $99 \%$ of radioactivity was excreted in the feces.

The objectives of the present study were to evaluate the safety of single oral dose E3030 in healthy Japanese male subjects, and to evaluate pharmacokinetics after single oral dose E3030 and food effect on pharmacokinetic profiles.

\section{Materials and Methods}

\subsection{Ethics}

This study was conducted at Sekino Clinical Pharmacology Clinic, Tokyo, Japan, in accordance with the ethical principles of the Declaration of Helsinki, Good Clinical Practice in Japan, and International Conference on Harmonization guidelines. The clinical study protocol and informed consent documents were approved by the Institutional Review Board of Sekino Clinical Pharmacology Clinic. Informed consent for all subjects was obtained in writing before conduct of any study-related procedures.

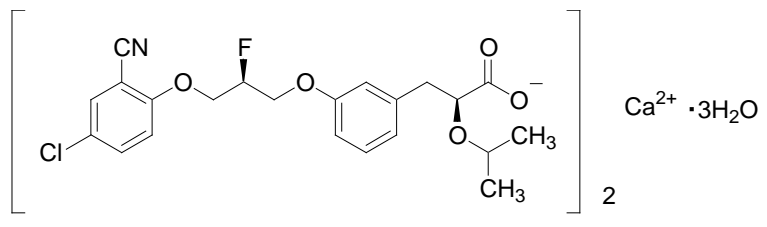

Figure 1. Molecular structure of E3030, monocalcium bis ((2S)-3-\{3-[(2S)-3-(4-chloro-2-cyanophenoxy)-2-fluoropropo xylphenyl\}-2-isopropoxypropionate) trihydrate.

\subsection{Study Design}

This study was conducted in a randomized, double-blind, placebo-controlled, single-dose manner, using ascending multiple doses of E3030. Different groups of eight subjects each were orally administered single doses of E3030 (0.5, 1, 2.5, 5, 10, 20, or $40 \mathrm{mg}, \mathrm{n}=6$ ) or placebo $(\mathrm{n}=2)$ after overnight fast of 10 hours. Of the group administered $10 \mathrm{mg}$ or placebo, seven subjects were additionally orally administered a single dose of $10 \mathrm{mg}$ E3030 $(n=5)$ or placebo $(n=2)$ at 30 minutes after starting normal breakfast (total energy, $800 \mathrm{kcal}$; fat, $28 \%$ ) following a 10-day or longer washout period. One subject administered $10 \mathrm{mg}$ in the fasted state did not proceed to the administration in the fed state, because the subject had creatine phosphokinase (CPK) elevation, probably due to living conditions during the washout period. The starting dose was selected based on the results of the toxicity studies in rats and dogs. The no-observed-adverse-effect level (NOAEL) in both species was $1 \mathrm{mg} / \mathrm{kg}$ based on the 4-week repeat-dose studies. The human equivalent dose (HED) of the NOAEL was calculated as $0.16 \mathrm{mg} / \mathrm{kg}$ (rats) and $0.54 \mathrm{mg} / \mathrm{kg}$ (dogs), in accordance with formulas described in the Food and Drug Administration (FDA) guidelines for estimating the safe starting dose in clinical trials [6]. The HED from the more sensitive species (rats) was converted to a dose of $9.6 \mathrm{mg}$ for a $60-\mathrm{kg}$ person. Using a safety factor of 10 , this resulted in an approximate dose of $1 \mathrm{mg}$. The starting dose was further lowered to $0.5 \mathrm{mg}$, representing a more conservative estimate. Doses were escalated stepwise from the starting dose of $0.5 \mathrm{mg}$ to $1,2.5,5,10,20 \mathrm{mg}$, and the maximum dose of $40 \mathrm{mg}$, using multiples of 2 to 2.5. The dose used to examine the effect of food on the pharmacokinetics of E3030 was $10 \mathrm{mg}$, which was expected to discriminate plasma concentrations in the fasted and fed states.

E3030 was supplied by Eisai Co., Ltd. E3030 was administered in tablets containing 0.1 , 1, or $5 \mathrm{mg}$ of E3030. Placebo tablets were visually matching tablets.

\subsection{Subjects}

Healthy Japanese male subjects between 20 and 39 years of age and with body mass index (BMI) of 18.5 to 25 $\mathrm{kg} / \mathrm{m}^{2}$ were eligible for participation in this study. Subjects were excluded if they had a known history of any significant drug or food allergy, a significant organ dysfunction, or any clinically significant deviation from normal in medical history, physical examination findings, vital signs, electrocardiogram, or laboratory test results. Subjects with gastrointestinal, hepatic, renal, respiratory, or cardiovascular diseases; congenital metabolic disorder; Gilbert's syndrome; deviations from normal reference 
range for liver enzymes at screening or baseline; a positive test result for hepatitis B surface antigen, hepatitis C antibody or human immunodeficiency virus; or alcohol or drug abuse (or a positive urine drug test result at screening) were excluded from participation. Subjects were excluded if they had a known history of any gastrointestinal surgery that could impact upon the absorption of study drug. Subjects were also excluded if they had experienced a weight change $>10 \%$ from screening to baseline. Furthermore, any subject were excluded who had received blood within 3 months or donated blood (400 mL within 3 months or $200 \mathrm{~mL}$ within 30 days of study start), or ingested any investigational medication within 4 months before study start. Subjects were prohibited from any prescription drugs or over-the-counter (OTC) acid controllers within 30 days prior to and during the study, or other OTC medications within 7 days prior to and during the study.

\subsection{Procedures}

Screening procedures, including medical history taking, physical examination, 12-lead electrocardiography (ECG), clinical laboratory evaluations, vital signs measurement, and urine drug screening, were performed within 30 days before study drug administration along with the assessment of inclusion/exclusion criteria. Eligible subjects were admitted to the study site on the day prior to dosing for baseline evaluations. Subjects were required to abstain from food and beverages, except water, for at least 10 hours prior to check-in. After the check-in evaluation was completed, subjects were provided with an appropriate meal(s); thereafter, they were required to fast (abstain from food and fluids, except water) overnight for at least 10 hours prior to drug administration the following day. Subjects took the study drug with $200 \mathrm{~mL}$ of water in fasted state, or at 30 minutes after starting breakfast when the effect of food on pharmacokinetics was investigated. Water was allowed ad libitum, except for 2 hours before dosing and 1 hour after dosing. Subjects were required to fast from food up to 4.5 hours after dosing. Lunch and subsequent meals were provided as per the regular meal schedule at the site. Subjects were to maintain an upright (seated or standing) position for at least 4.5 hours following administration of study medication.

\subsection{Pharmacokinetic Assessments}

\subsubsection{Sample Collection}

Blood samples for measuring plasma E3030 concentrations were collected at 0 (pre-dose), $0.33,0.67,1,1.5,2$, $3,4,6,8,10,12,24,36,48,60,72$, and 96 hours after administration of the study medication. Blood samples (4 $\mathrm{mL}$ each) were collected from a cutaneous vein in the forearm into a sodium-heparinized tube and placed on ice immediately after mixing by inversion at least 8 to 10 times. Within 2 hours of collection, samples were centrifuged $\left(4^{\circ} \mathrm{C}\right.$ at $2500 \mathrm{rpm}$ for 15 minutes) to obtain plasma, and at least $1.5 \mathrm{~mL}$ of plasma was transferred to a sample tube and stored at $-70^{\circ} \mathrm{C}$.

\subsubsection{Bioanalytical Methods}

Bioanalysis was performed by the Bioanalysis Section, Clinical Research Center at Eisai Co., Ltd (Tokyo, Japan). Plasma samples in the study were analyzed for the quantitative determination of E3030 by a validated liquid chromatographic-tandem mass spectrometric (LC/MS/MS) method. The method is based on solid-phase extraction using Empore $^{\mathrm{TM}}$ extraction disk plates (3 M, St. Paul, $\mathrm{MN}$ ) in the 96-well format, with $2.5 \mu \mathrm{L}$ eluent samples injected into the LC/MS/MS system. Human plasma samples were analyzed in 19 batches. Each batch typically consisted of a calibration curve generated by use of $0.1,0.25,0.5,2.5,5,20,50$, and $100 \mathrm{ng} / \mathrm{mL}$ of E3030, one blank plasma sample containing internal standard (IS), one blank plasma sample, quality control (QC) samples at three concentrations each in duplicate, and 72 clinical samples from four subjects.

Sample preparation before loading onto extraction disk plate was conducted on ice bath. IS (ER-228063-00, (2S)-3-\{3-[2-(4-fluorophenoxy)ethoxy]phenyl\}-2-isopro poxypropionic acid) solution (20 $\mu \mathrm{L}$ each) was accurately added to $220 \mu \mathrm{L}$ of clinical plasma samples (previously, $20 \mu \mathrm{L}$ each of $50 \% \mathrm{CH}_{3} \mathrm{OH} / \mathrm{H}_{2} \mathrm{O}$ had been accurately added to $0.20 \mathrm{~mL}$ of clinical samples), calibration samples and QC samples, and then the samples were mixed well. Afterwards, $200 \mu \mathrm{L}$ of distilled water and $100 \mu \mathrm{L}$ of $1 \mathrm{~mol} / \mathrm{L} \mathrm{HCl}$ (both previously placed on ice bath) were added, and the samples were mixed well. The extraction disk plate had been previously conditioned with $100 \mu \mathrm{L}$ $\mathrm{CH}_{3} \mathrm{OH}$ followed by addition of $300 \mu \mathrm{L}$ of distilled water and aspiration for 5 seconds. Samples were loaded onto the extraction disk plate and aspirated to waste. The extraction disk plate was washed with $400 \mu \mathrm{L}$ of distilled water 3 times, $200 \mu \mathrm{L}$ of $20 \% \mathrm{CH}_{3} \mathrm{CN} / \mathrm{H}_{2} \mathrm{O}$ once, and $200 \mu \mathrm{L}$ of $40 \% \mathrm{CH}_{3} \mathrm{CN} / \mathrm{H}_{2} \mathrm{O}$ once by aspiration. Analytes were eluted with $80 \mu \mathrm{L}$ of $80 \% \mathrm{CH}_{3} \mathrm{CN} / \mathrm{H}_{2} \mathrm{O}$ to a 96-well collection plate by centrifugation at $1500 \mathrm{rpm}$ (252 g) for 3 minutes twice. $\mathrm{CH}_{3} \mathrm{COOH}-\mathrm{CH}_{3} \mathrm{COONH}_{4}$ buffer ( $50 \mu \mathrm{L}, 50 \mathrm{mmol} / \mathrm{L}$, pH5.0) was added to the extracts, the samples were mixed well, and a $2.5 \mu \mathrm{L}$ portion was injected into the LC/MS/MS system.

E3030 and IS were separated by liquid chromatography using an Inertsil ODS-3 column (3.0 mm i.d. x 75 $\mathrm{mm}$ ) (GL sciences, Inc., Tokyo, Japan) with mobile phase consisting of $7.5 \mathrm{mmol} / \mathrm{L} \mathrm{CH}_{3} \mathrm{COONH}_{4}-\mathrm{H}_{2} \mathrm{O}-$ $\mathrm{CH}_{3} \mathrm{CN}(10: 140: 450, \mathrm{v} / \mathrm{v} / \mathrm{v})$ at a flow rate of $0.3 \mathrm{~mL} / \mathrm{min}$. 
A PE Sciex API 4000 mass spectrometer equipped with negative electrospray ionization was used in multiple reaction monitoring mode. Low and unit resolution were used for Q1 and Q3 detection, respectively. The precursor to product ion transitions of $\mathrm{m} / \mathrm{z} 434.2 \rightarrow 152.1$ for E3030, and $\mathrm{m} / \mathrm{z} 361.1 \rightarrow 119.3$ for IS, were monitored.

The correlation coefficients of calibration curves ranged from 0.9988 to 0.9999 , and the accuracy of calibration samples ranged from $-1.5 \%$ to $3.7 \%$ at the lower limit of quantification (LLOQ), and from $-6.1 \%$ to $7.9 \%$ for those values other than the LLOQ. The accuracy for E3030 in human plasma was measured at 3 QC sample concentrations. The accuracy at $0.300 \mathrm{ng} / \mathrm{mL}, 3.00 \mathrm{ng} / \mathrm{mL}$, and $80.0 \mathrm{ng} / \mathrm{mL}$ ranged from $-8.7 \%$ to $10.7 \%$, from $-11.4 \%$ to $8.5 \%$ ( $-9.8 \%$ to $7.0 \%$ except for one sample of the first batch at the $0.5 \mathrm{mg}$ dose), and from $-19.3 \%$ to $7.0 \%$, respectively. The data for calibration curves and QC samples concerning in-study validation showed good analytical performance.

\subsubsection{Pharmacokinetic Analysis}

Pharmacokinetic parameters were calculated from plasma concentrations of E3030 as a free base by model-independent analysis using WinNonlin Professional version 4.1 (Pharsight Corp., Mountain View, CA). For calculation of pharmacokinetic parameters, measured concentration values less than the LLOQ $(0.10 \mathrm{ng} / \mathrm{mL})$ were defined as zero. All calculations of the plasma pharmacokinetics were based on actual sampling times. Maximum plasma concentrations $\left(\mathrm{C}_{\max }\right)$ observed and time to $\mathrm{C}_{\max }\left(\mathrm{t}_{\max }\right)$ were obtained directly from experimental data. The area under plasma concentration-time curve from 0 time extrapolated to infinite time (AUC) was determined as follows:

$$
\mathrm{AUC}=\mathrm{AUC}_{0-\mathrm{t}}+\text { last observed concentration } / \lambda_{\mathrm{z}}
$$

The area under the plasma concentration-time curve from 0 time to time of last quantifiable point $\left(\mathrm{AUC}_{0-\mathrm{t}}\right)$ was calculated using the linear-log trapezoidal method. The terminal phase rate constant $\left(\lambda_{z}\right)$ was estimated by linear regression versus time curve. The $t_{1 / 2}$ was calculated as $\ln (2) / \lambda_{z}$; apparent clearance (CL/F), as dose/ AUC; $V_{\mathrm{z}} / \mathrm{F}$, as $\mathrm{CL} / \mathrm{F} / \lambda_{\mathrm{z}}$.

The dose-proportionality of $\mathrm{C}_{\max }$ and AUC obtained from model-independent analysis was assessed both visually and using a power model $\left(\mathrm{Y}=\alpha \mathrm{X}^{\beta}\right.$; $\mathrm{X}$, Dose; $\mathrm{Y}$, $\mathrm{C}_{\max }$ or AUC). Dose proportionality was assessed based on whether 95\% confidence intervals (CIs) of $\beta$ lay within the range from 0.7 to 1.3 [7].

To assess the effect of food on the pharmacokinetics of E3030, $\mathrm{C}_{\max }$ and AUC in the fed state were compared with those in the fasted state, with those in the fasted state used as the reference. Evaluation was performed on subjects for whom pharmacokinetic data from fasted and fed states were available. The geometric mean ratio (fasted/fed) and its $90 \% \mathrm{CI}$ were calculated for the $\mathrm{C}_{\max }$ and AUC. Food effect on the pharmacokinetics of E3030 was assessed based on whether $90 \%$ CIs of ratios of AUC and $\mathrm{C}_{\max }$ lay within the range from 0.80 to 1.25 [8].

\subsection{Safety Assessments}

The following data were collected during the study to assess safety: physical examination findings, vital signs (blood pressure, pulse rate, respiratory rate, and body temperature), body weight, 12-lead ECGs, and clinical laboratory parameters (hematology, biochemistry and urinalysis). In the case of clinically significant abnormal results, that evaluation was to be repeated until the values were within an acceptable or normal range. Adverse events were to be followed to resolution.

The numbers of subjects with adverse events were tabulated. For clinical laboratory parameters (except urinalysis), vital signs, body weight, and 12-lead ECG parameters, the values measured and changes from baseline at each time were summarized by dose using descriptive statistics. For urinalysis, cross tables were prepared.

\section{Results}

\subsection{Subject Demographics}

A total of 56 healthy Japanese male subjects were enrolled. Subject demographics across dose groups were similar (Table 1). The mean age of enrolled subjects was $24.7 \pm 3.8$ years (mean \pm standard deviation; range, 20 35 years), with an average BMI of $21.38 \pm 1.76 \mathrm{~kg} / \mathrm{m}^{2}$ (range, $18.8-24.5 \mathrm{~kg} / \mathrm{m}^{2}$ ). Due to CPK elevation (501 $\mathrm{IU} / \mathrm{L}$ ) at the check-in day after a washout period, one of six subjects administered $10 \mathrm{mg}$ did not proceed to the fed state to avoid the influence on safety assessment. All other subjects completed the study.

\subsection{Safety and Tolerability}

No deaths or serious adverse events were reported following single oral doses of 0.5 to $40 \mathrm{mg}$ E3030. Adverse events observed in this study are listed in Table 2. No subjective symptoms, objective findings, or abnormal changes in vital signs were observed. Although abnormal changes occurred in laboratory parameters, i.e. increases of amylase, total bilirubin, CPK, aspartate aminotransferase (AST), and TG, in five of six subjects, all changes were mild and recovered spontaneously. One subject showed an abnormal 12-lead ECG finding (supraventricular extrasystole). This episode was mild, not associated with subjective symptoms or objective findings, and resolved spontaneously. All adverse events were mild and 
Table 1. Demographic characteristics of the study population.

\begin{tabular}{cccccc}
\hline E3030 dose & No. of subjects & Age (years) & Height $(\mathrm{cm})$ & Body weight $(\mathrm{kg})$ & BMI $\left(\mathrm{kg} / \mathrm{m}^{2}\right)$ \\
\hline $0.5 \mathrm{mg}$ & 6 & $23.3 \pm 2.9$ & $168.88 \pm 7.36$ & $61.68 \pm 8.84$ & $21.58 \pm 2.28$ \\
$1 \mathrm{mg}$ & 6 & $25.3 \pm 4.2$ & $171.18 \pm 5.00$ & $60.25 \pm 5.50$ & $20.53 \pm 0.84$ \\
$2.5 \mathrm{mg}$ & 6 & $25.0 \pm 3.9$ & $168.70 \pm 7.56$ & $64.43 \pm 7.25$ & $22.60 \pm 1.53$ \\
$5 \mathrm{mg}$ & 6 & $25.7 \pm 3.3$ & $174.98 \pm 7.22$ & $64.63 \pm 7.84$ & $21.05 \pm 1.45$ \\
$10 \mathrm{mg}$ & 6 & $29.0 \pm 3.7$ & $173.43 \pm 6.60$ & $67.62 \pm 3.97$ & $22.52 \pm 1.19$ \\
$20 \mathrm{mg}$ & 6 & $24.3 \pm 4.9$ & $176.30 \pm 5.44$ & $69.43 \pm 7.99$ & $22.30 \pm 2.01$ \\
$40 \mathrm{mg}$ & 6 & $23.0 \pm 1.7$ & $172.60 \pm 6.06$ & $58.13 \pm 3.68$ & $19.52 \pm 0.44$ \\
All E3030-treated & 42 & $25.1 \pm 3.9$ & $172.30 \pm 6.62$ & $63.74 \pm 7.25$ & $21.44 \pm 1.76$ \\
All placebo-treated & 14 & $23.4 \pm 3.2$ & $169.28 \pm 5.96$ & $60.73 \pm 4.99$ & $21.21 \pm 1.79$ \\
All subjects & 56 & $24.7 \pm 3.8$ & $171.54 \pm 6.54$ & $62.99 \pm 6.84$ & $21.38 \pm 1.76$ \\
\hline
\end{tabular}

BMI, body mass index. Mean \pm standard deviation.

Table 2. Summary of adverse events after single oral dose of E3030.

\begin{tabular}{|c|c|c|c|c|c|c|c|c|c|}
\hline & \multicolumn{9}{|c|}{ E3030 dose } \\
\hline & Placebo & $0.5 \mathrm{mg}$ & $1 \mathrm{mg}$ & $2.5 \mathrm{mg}$ & $5 \mathrm{mg}$ & $10 \mathrm{mg}$, fasted & $10 \mathrm{mg}$, fed & $20 \mathrm{mg}$ & $40 \mathrm{mg}$ \\
\hline & $(n=16)$ & $(n=6)$ & $(n=6)$ & $(n=6)$ & $(\mathrm{n}=6)$ & $(\mathrm{n}=6)$ & $(n=5)$ & $(n=6)$ & $(n=6)$ \\
\hline Supraventricular extrasystole & 0 & 0 & 0 & 0 & 0 & 0 & 0 & 1 & 0 \\
\hline AST increased & 0 & 0 & 0 & 0 & 0 & 0 & 0 & 0 & 1 \\
\hline Amylase increased & 0 & 1 & 0 & 0 & 0 & 0 & 0 & 0 & 0 \\
\hline CPK increased & 0 & 0 & 0 & 0 & 0 & $1^{\mathrm{a}}$ & 0 & 0 & 1 \\
\hline TG increased & 0 & 0 & 0 & 0 & 0 & 0 & 0 & 0 & $1^{\mathrm{b}}$ \\
\hline Total bilirubin increased & 0 & 1 & 0 & 0 & 0 & 0 & 0 & 0 & 0 \\
\hline
\end{tabular}

AST, aspartate aminotransferase; CPK, creatine phosphokinase; TG, triglyceride. (a) Judged as not related to the study drug, because CPK elevation developed 20 days after administration of $10 \mathrm{mg}$ in the fasted state: considered probably due to living conditions in the washout period. (b) Judged as not related to the study drug, because TG elevation had developed by outpatient visit 6 days after study drug administration: considered probably due to influence of meals after check-out.

transient, and there was no dose-dependent increase in any adverse event. The maximum tolerated dose was not reached, because the highest dose of $40 \mathrm{mg}$ and therefore all other doses were considered tolerated. Based on the above results, there were no concerns about the safety or tolerability of E3030 at doses between 0.5 and $40 \mathrm{mg}$.

\subsection{Pharmacokinetics}

Pharmacokinetic profiles of E3030 were assessed in 42 healthy adult male subjects receiving a single oral dose of E3030 (0.5, 1, 2.5, 5, 10, 20, or $40 \mathrm{mg})$ under fasted conditions. Mean pharmacokinetic profiles after the single dose are presented in Figure 2, and E3030 single-dose pharmacokinetic parameters are provided in Table 3. E3030 was rapidly absorbed after dosing, with $t_{\max }$ of 1 - 1.75 hours; thereafter, concentrations declined nearly biexponentially with a terminal half-life of 18.4 23.8 hours. Both $\mathrm{C}_{\max }$ and AUC showed dose-proportional increases. Following administration of 0.5 to 40 mg E3030, mean $\mathrm{C}_{\max }$ increased from 58 to $4608 \mathrm{ng} / \mathrm{mL}$, and mean AUC increased from 887 to 68,994 ng. hr/mL. Mean CL/F ranged between 601 and $716 \mathrm{~mL} / \mathrm{hr}$. Mean $\mathrm{CL} / \mathrm{F}$ and $\mathrm{V}_{\mathrm{z}} / \mathrm{F}$ showed almost same value over the studied dose range.

Dose-proportionality for $\mathrm{C}_{\max }$ and AUC obtained from model-independent analysis was assessed both visually and using a power model $\left(\mathrm{Y}=\alpha \mathrm{X}^{\beta} ; \mathrm{X}\right.$, Dose; $\mathrm{Y}, \mathrm{C}_{\max }$ or AUC). Plots of individual $\mathrm{C}_{\max }$ and AUC against dose are presented in Figure 3. The plots illustrate dose-proportional increases in $C_{\max }$ and AUC. Point estimates of $\beta$ in $\mathrm{C}_{\max }$ and AUC were 0.976 and 0.985 , respectively, 


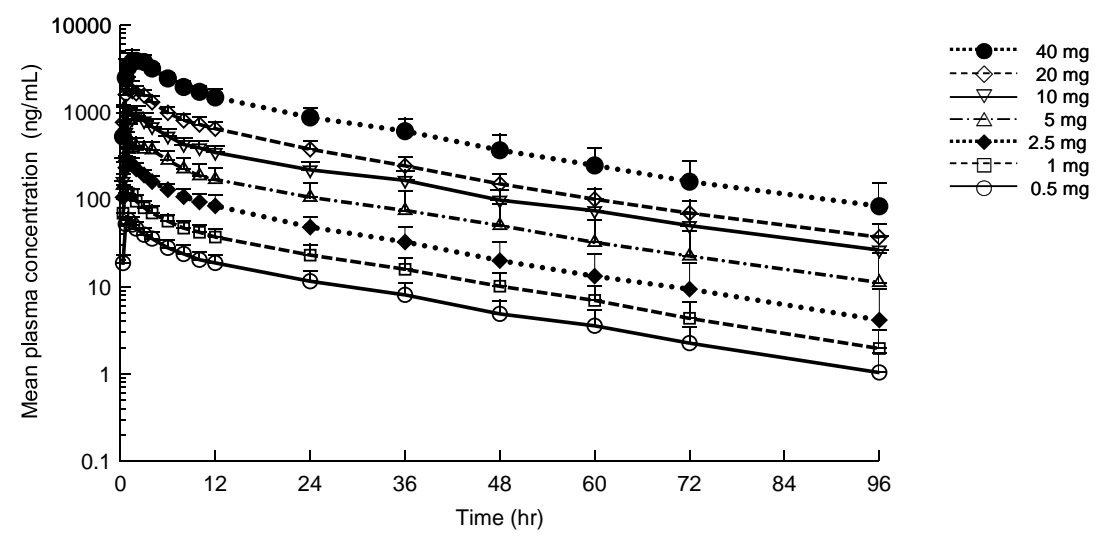

Figure 2. Mean plasma E3030 concentrations after single oral dose of E3030 (0.5 - $40 \mathrm{mg})$ in healthy adult male subjects under fasted conditions. Each plotted point represents mean value and standard deviation (six subjects per group).

Table 3. Summary of pharmacokinetic parameters obtained by model-independent analysis.

\begin{tabular}{lccccccc}
\hline \multirow{2}{*}{ Parameter } & \multicolumn{7}{c}{ E3030 dose } \\
\cline { 2 - 8 } & $0.5 \mathrm{mg}$ & $1 \mathrm{mg}$ & $2.5 \mathrm{mg}$ & $5 \mathrm{mg}$ & $10 \mathrm{mg}$ & $20 \mathrm{mg}$ & $40 \mathrm{mg}$ \\
\hline $\mathrm{C}_{\max }(\mathrm{ng} / \mathrm{mL})$ & $58.3 \pm 12.4$ & $123.0 \pm 17.0$ & $282.7 \pm 41.4$ & $467.2 \pm 88.1$ & $1066.7 \pm 162.0$ & $2048.4 \pm 450.5$ & $4608.0 \pm 788.3$ \\
$\mathrm{t}_{\max }(\mathrm{hr})$ & $1(0.67-1.00)$ & $1(0.67-1.50)$ & $1(0.67-1.50)$ & $1.75(1.00-4.00)$ & $1.5(1.00-3.00)$ & $1(0.67-3.00)$ & $1.5(0.67-3.00)$ \\
$\mathrm{AUC}(\mathrm{ng} \cdot \mathrm{hr} / \mathrm{mL})$ & $886.8 \pm 266.1$ & $1782.0 \pm 498.9$ & $3877.3 \pm 1457.1$ & $8320.4 \pm 4178.1$ & $17163.4 \pm 3720.6$ & $29793.1 \pm 6516.7$ & $68933.8 \pm 21132.0$ \\
$\mathrm{t}_{1 / 2}(\mathrm{hr})$ & $19.7 \pm 3.3$ & $18.9 \pm 2.8$ & $18.4 \pm 5.9$ & $20.6 \pm 6.1$ & $22.7 \pm 3.2$ & $23.8 \pm 3.7$ & $19.5 \pm 4.8$ \\
$\mathrm{CL} / \mathrm{F}(\mathrm{mL} / \mathrm{hr})$ & $612 \pm 199$ & $601 \pm 177$ & $708 \pm 214$ & $716 \pm 310$ & $611 \pm 159$ & $700 \pm 157$ & $629 \pm 197$ \\
$\mathrm{~V}_{\mathrm{z} / \mathrm{F}}(\mathrm{L})$ & $16.7 \pm 3.2$ & $16.0 \pm 3.9$ & $17.6 \pm 2.5$ & $20.0 \pm 8.2$ & $19.5 \pm 3.3$ & $23.6 \pm 4.2$ & $16.8 \pm 2.7$ \\
\hline
\end{tabular}

$\mathrm{C}_{\max }$, maximum observed concentration; $\mathrm{t}_{\max }$, time to $\mathrm{C}_{\max }$; AUC, area under the concentration-time curve from 0 time extrapolated to infinite time; $\mathrm{t}_{1 / 2}$, terminal elimination phase half-life; CL/F, apparent clearance; $V_{z} / F$, apparent volume of distribution at terminal phase. Mean \pm standard deviation, except $t_{\text {max }}$. $t_{\text {max }}$ : median (minimum-maximum). Six subjects per group.

indicating that both values were approximately 1 . The $95 \%$ CIs of $\beta$ for $\mathrm{C}_{\max }$ and AUC were $0.936-1.016$ and 0.920 - 1.050, respectively. These results suggest that both $C_{\max }$ and AUC following a single oral dose of E3030 (0.5 - 40 $\mathrm{mg}$ ) increase dose-proportionally.

Food effect on pharmacokinetic profiles of E3030 in five healthy adult male subjects was assessed by comparison of plasma pharmacokinetic profiles after a single oral dose of $10 \mathrm{mg}$ under fasted condition and those at 30 minutes after start of a meal. The mean pharmacokinetic profiles under both conditions are shown in Figure 4, and intra-subject pharmacokinetic parameters are provided in Table 4. Plasma E3030 concentrations under fed condition were lower than those under fasted condition; otherwise, biexponential elimination patterns were similarly observed. The ratio of geometric mean values (fed/fasted) of $\mathrm{C}_{\max }$ and AUC were 0.803 and 0.913 , respectively. In addition, the $95 \%$ CIs of these were 0.738 0.874 and 0.809 - 1.031, respectively. These results suggest that food effect on pharmacokinetics of E3030 appeared to be not significant considering the equivalent
AUC despite a decrease in $\mathrm{C}_{\max }$ of approximately $20 \%$.

\section{Discussion}

Subjective symptoms, objective findings, and abnormal changes in vital signs were not observed after single doses of E3030 up to $40 \mathrm{mg}$. Abnormal changes in laboratory values for which a causal relationship with E3030 could not be excluded were amylase increased in one subject in the $0.5 \mathrm{mg}$ group, total bilirubin increased in another subject in the same group, and CPK increased and AST increased in one subject in the $40 \mathrm{mg}$ group. Because the subject with amylase increased in the $0.5 \mathrm{mg}$ group did not have subjective symptoms or objective findings suggestive of pancreatitis and did not have lipase increased as well as white blood cell count increased, the mechanism of his amylase increase was not explored but was considered to be specific to the relevant subject. Regarding the case of total bilirubin increased (from $1.0 \mathrm{mg} / \mathrm{dL}$ at baseline to $1.8 \mathrm{mg} / \mathrm{dL}$ at 24 hours after dose), although that increase was regarded as abnormal, 


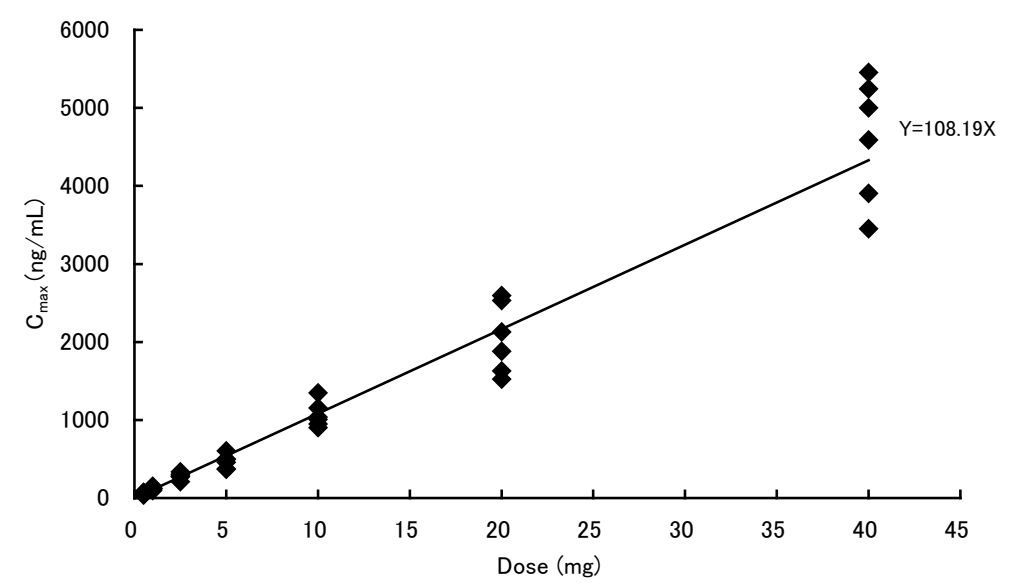

(a)

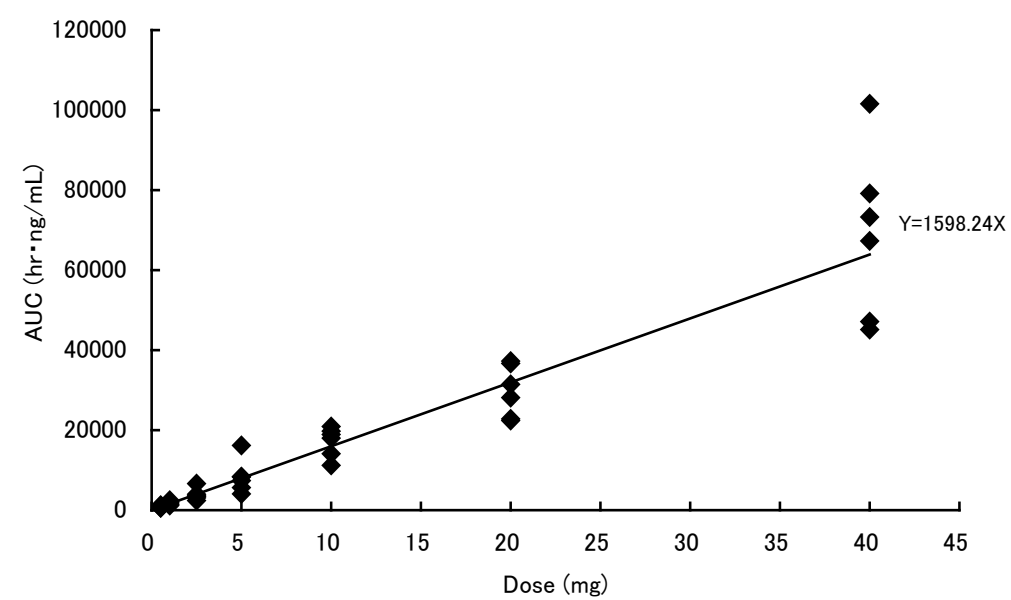

(b)

Figure 3. Relationships between dose and (a) $C_{\max }$ or (b) AUC after single oral dose of E3030 (0.5 - 40 mg) in healthy adult male subjects under fasted conditions. Each point: individual value. Solid line: linear regression model from a method of least squares.

Table 4. Summary of intra-subject pharmacokinetic parameters obtained by model-independent analysis after single oral dose of $10 \mathrm{mg}$ E3030 in the fasted and fed states.

\begin{tabular}{lcc}
\hline \multirow{2}{*}{ Parameter } & \multicolumn{2}{c}{ E3030 10-mg dosing } \\
\cline { 2 - 3 } & Fasted & Fed \\
\hline $\mathrm{C}_{\max }(\mathrm{ng} / \mathrm{mL})$ & $1099.1 \pm 157.8$ & $888.7 \pm 168.5$ \\
$\mathrm{t}_{\max }(\mathrm{hr})$ & $1(1.00-2.00)$ & $1.5(0.67-2.00)$ \\
$\mathrm{AUC}(\mathrm{ng} \cdot \mathrm{hr} / \mathrm{mL})$ & $16647.6 \pm 3912.6$ & $15598.4 \pm 5370.6$ \\
$\mathrm{t}_{1 / 2}(\mathrm{hr})$ & $22.5 \pm 3.5$ & $24.9 \pm 5.5$ \\
\hline
\end{tabular}

$\mathrm{C}_{\max }$, maximum observed concentration; $\mathrm{t}_{\max }$, time to $\mathrm{C}_{\max }$; AUC, area under the concentration-time curve from 0 time extrapolated to infinite time; $\mathrm{t}_{1 / 2}$, terminal elimination phase half-life. Mean \pm standard deviation, except $t_{\max }$. $\mathrm{t}_{\max }$ : median (minimum-maximum). Five subjects per group.

the total bilirubin of this subject was already $1.4 \mathrm{mg} / \mathrm{dL}$ at screening; therefore it was considered that the subject might be a person normally with high total bilirubin levels. In addition, amylase increased and total bilirubin increased were observed in one subject in the $0.5 \mathrm{mg}$ group, but such increases were not observed in the 1 to $40 \mathrm{mg}$ groups. And no increase in incidence was observed with an escalating dose of E3030. Therefore, these increases might have occurred incidentally. Regarding CPK increased (96 hours after dose) and AST 


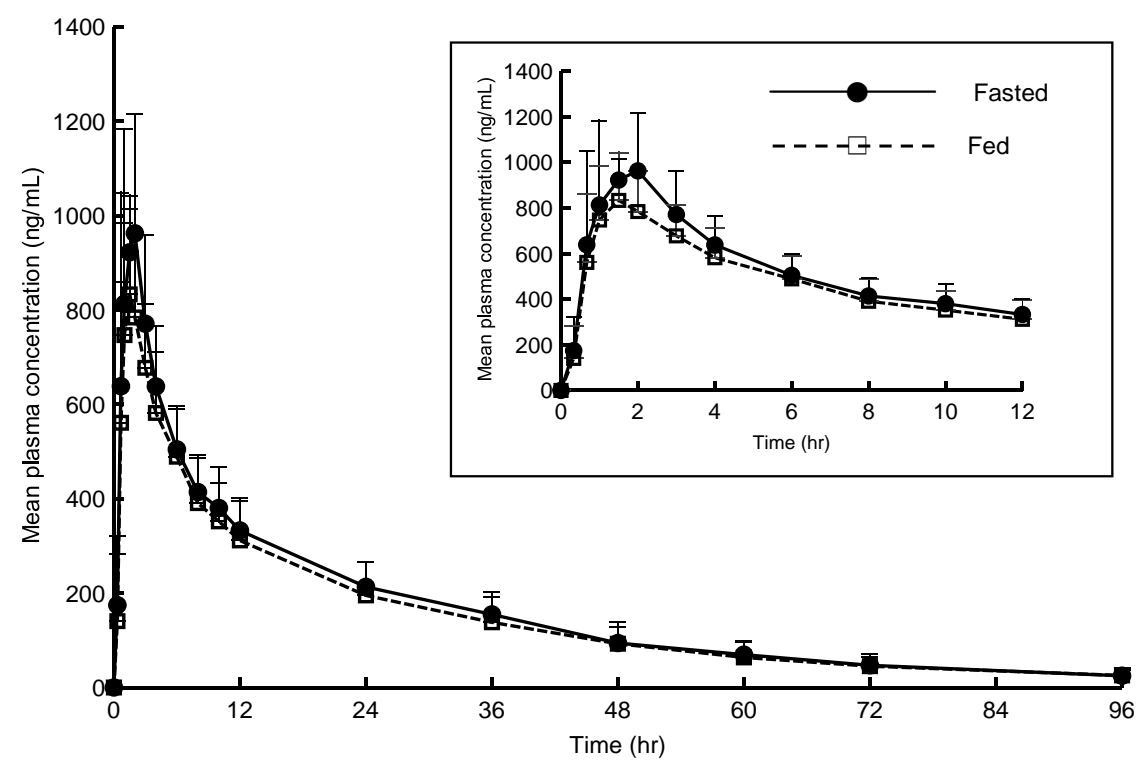

Figure 4. Mean plasma E3030 concentrations after single oral dose of E3030 (10 $\mathrm{mg})$ in healthy adult male subjects under fasted and fed conditions. Each plotted point represents mean value and standard deviation (five subjects per group).

increased (6 days after dose) in a subject in the $40 \mathrm{mg}$ group, although a causal relationship with E3030 could not be excluded in view of the time after administration of the study drug, both values had returned to the reference range within 10 days after E3030 administration without any treatment. The AUC of this subject was $101545.76 \mathrm{ng} \cdot \mathrm{hr} / \mathrm{mL}$, approximately 1.47 times as high as the mean AUC (68933.835 $\mathrm{ng} \cdot \mathrm{hr} / \mathrm{mL})$ for the six subjects who received $40 \mathrm{mg}$ of E3030; however, it was considered difficult to determine on the basis of this one subject only whether a relationship existed between increase of CPK/AST and plasma concentration of E3030. As abnormal change in 12-lead ECG, supraventricular extrasystoles were observed in one subject in the $20 \mathrm{mg}$ group. Since the QRS wave was normal and there were no subjective or objective findings, this change was not considered clinically significant. Supraventricular extrasystoles are often observed in healthy persons, and the reported extrasystoles might have been noticed during frequent measurements in the clinical study. Otherwise, supraventricular extrasystoles, often occurring during rest, might have developed during hospital stay in the clinical study. Although a causal relationship with E3030 could not be excluded in view of the time after administration of the study drug, the extrasystoles remitted without any treatment. All adverse events were mild and transient, and there was no dose-dependent increase in any adverse event. The above results suggest that there are no problems with safety or tolerability when E3030 is administered in a single dose up to $40 \mathrm{mg}$.

E3030 was developed for improving the control of blood glucose in diabetic patients by improving their insulin resistance; therefore, the study drug is less likely to induce hypoglycemia, as compared with other drugs, including sulfonylurea agents with insulin secretionstimulating activity. No sign or symptom of hypoglycemia was observed during this study. E3030, a PPAR $\alpha / \gamma$ dual agonist, can cause such adverse drug reactions (ADRs) as those observed after administration of PPAR $\alpha$ or PPAR $\gamma$ agonists. Thiazolidine derivatives, which are $\operatorname{PPAR} \gamma$ agonists, have been reported to cause ADRs including oedema, anaemia and weight gain [9]. In the SYNCHRONY study, a phase II study of the PPAR $\alpha / \gamma$ dual agonist aleglitazar, oedema, haemodilution, and weight gain occurred in a dose-dependent manner [10]. But there were no findings suggesting these ADRs during this study, and the possibility that these ADRs might occur during this single-dose study was considered to be lower than in actual clinical settings. In addition, fibrate drugs, which are PPAR $\alpha$ agonists, have been reported to cause liver transaminases increased, white blood cell count decreased, anemia, gallbladder disorders, and rhabdomyolysis [11-13]. In the current study, although CPK increased and AST increased, both considered to originate from muscle, in a subject in the $40 \mathrm{mg}$ group were mild and transient, a relationship with rhabdomyolysis could not be excluded. These ADRs related to $\operatorname{PPAR} \gamma$ - or $\operatorname{PPAR} \alpha$-activating activities should also be monitored carefully in future studies.

Pharmacokinetic profiles after a single oral dose of E3030 were assessed in healthy adult male volunteers. Metabolites of E3030 were not measured in this study, because only a glucuronide of E3030 was identified as a major metabolite in non-clinical studies, and because it 
was supposed that the glucuronide did not have pharmacological activity. E3030 was rapidly absorbed and was eliminated nearly biexponentially after reaching $C_{\max }$. Among pharmacokinetic parameters calculated using a model-independent method, the mean $t_{1 / 2}$ was approximately 20 hours, remaining relatively constant over the dose range examined in this study. Consequently, from a pharmacokinetic viewpoint, E3030 was considered a drug that can be administered once daily. Similarly, CL/F and $\mathrm{V}_{\mathrm{Z}} / \mathrm{F}$ also remained nearly constant regardless of dose levels. Clearance and distribution volume after oral administration are affected by bioavailability (F), so that it is difficult to describe the two parameters exactly. However, assuming that $\mathrm{F}$ equals 1 , the clearance (CL) is smaller than the hepatic blood flow of $1450 \mathrm{~mL} / \mathrm{min}(87,000$ $\mathrm{mL} / \mathrm{hr})$ [14], and distribution volume $\left(\mathrm{V}_{\mathrm{z}}\right)$ is almost equivalent to the extracellular fluid volume, suggesting low levels of both parameters. The plasma concentration profile of administered E3030 exhibited bimodal peaks in non-clinical pharmacokinetic studies (rat and dog), suggesting the presence of enterohepatic circulation. In this clinical study, however, the plasma concentration changed with prominent bimodal peaks, supporting the absence of enterohepatic circulation. After a single oral administration of radiolabeled E3030 to rats and dogs, approximately $99 \%$ of radioactivity was excreted in the feces. The main elimination route is supposed to be fecal excretion also in human, however, further clinical pharmacokinetic study using radiolabeled E3030 is needed to evaluate more detailed pharmacokinetics of E3030.

Investigation of the relationship between $\mathrm{C}_{\max }$ or AUC and the dose levels showed that $\mathrm{C}_{\max }$ and AUC values from each subject tended to be distributed along a straight line passing through origin. After formulating a power curve $\mathrm{Y}=\alpha \mathrm{X}^{\beta}$ between dose levels $(\mathrm{X})$ and $\mathrm{C}_{\max }$ or AUC (Y), we fitted a linear regression equation $\log (\mathrm{Y})$ $=\log (\alpha)+\beta \times \log (\mathrm{X})$ by taking the $\log$ of both sides. The calculated point estimates for $\beta$ of $\mathrm{C}_{\max }$ and AUC were found to be 0.976 and 0.985 respectively, both being close to 1 , and the $95 \%$ CIs for $\beta$ were $0.936-1.016$ and $0.920-1.050$, respectively. It has been reported that if the $95 \% \mathrm{CI}$ for $\beta$ falls into the range of $0.7-1.3$, the relation can be regarded as linear [7]. The results from this study lay within this range, suggesting that E3030 showed a good linear pharmacokinetic profile when orally administered in a single dose under fasted condition within the dose range of 0.5 to $40 \mathrm{mg}$ examined in this study.

In this study, food effect on plasma pharmacokinetic profiles of E3030 was investigated exploratively in five healthy adult male volunteers. Instead of the crossover method used in general, a parallel comparative method was employed, because the investigation was conducted in a multi-step dose-escalation manner in the present study. The meal provided to subjects was a normal diet with total calories of approximately $800 \mathrm{kcal}$ (fat, approximately 28\%). Review of the parameters for each subject revealed that $C_{\max }$ was reduced in all five subjects when the drug was administered in the fed state, but the change in AUC due to food effect did not show a specific tendency. The ratios of geometric mean values (fed/ fasted) for $\mathrm{C}_{\max }$ and AUC were 0.803 and 0.913 , respectively, and the $90 \%$ CI of the ratio for AUC met a criterion for determining the absence of food effect [8], while that for $\mathrm{C}_{\max }$ did not meet this criterion. Although $\mathrm{C}_{\max }$ was reduced by around $20 \%$ after administration under fed condition, AUC under fed condition was the same as under fasted condition, suggesting that the food effect on the pharmacokinetics of E3030 was not significant, and there was no necessity to restrict the administration method of this drug to before meal or after meal.

\section{Conclusion}

In conclusion, no concerns regarding the safety or tolerability were noted after a single oral dose of E3030 in the dose range between 0.5 and $40 \mathrm{mg}$. Both $\mathrm{C}_{\max }$ and AUC of E3030 increased dose-dependently, indicating that E3030 has a favorably linear pharmacokinetic profile. The mean $t_{1 / 2}$ of E3030 was approximately 20 hours, and pharmacokinetic profiles indicated q.d. administration. Since food effect on pharmacokinetics of E3030 was not significant, no limitation to before-meal or after-meal dosing was suggested.

\section{Acknowledgements}

The authors would like to thank the volunteers who participated in this first-in-man study, and staff at Sekino Clinical Pharmacology Clinic. The authors also thank the Eisai study team: Hideyuki Samma and Yuko Kurihara for measurement of plasma E3030 concentrations; Rikako Kuroda for clinical data management; Hideaki Miyagishi for statistical analysis; Sari Shiba for pharmacokinetic analysis; and Dr Hirohiko Daimon for study monitoring. The authors would also like to express our gratitude to Dr Yukio Horai for his helpful advice in drafting the manuscript.

\section{Funding}

This study was sponsored by Eisai Co., Ltd. Dr. Hasegawa was the director of the study site. Dr. Ohnishi was a paid consultant to Eisai and other pharmaceutical companies.

\section{REFERENCES}

[1] J. A. Dormandy, B. Charbonnel, D. J. Eckland, et al., "Secondary Prevention of Macrovascular Events in $\mathrm{Pa}$ - 
tients with Type 2 Diabetes in the PROactive Study (PRO Spective Pioglit Azone Clinical Trial in MacroVascular Events): A Randomised Controlled Trial," Lancet, Vol. 366, No. 9493, 2005, pp. 1279-1289. http://dx.doi.org/10.1016/S0140-6736(05)67528-9

[2] A. Keech, R. J. Simes, P. Barter, et al., "Effects of LongTerm Fenofibrate Therapy on Cardiovascular Events in 9795 People with Type 2 Diabetes Mellitus (the FIELD Study): Randomised Controlled Trial,” Lancet, Vol. 366, No. 9500, 2005, pp. 1849-1861. http://dx.doi.org/10.1016/S0140-6736(05)67667-2

[3] ACCORD Study Group, H. N. Ginsberg, M. B. Elam, L. C. Lovato, et al., "Effects of Combination Lipid Therapy in Type 2 Diabetes Mellitus," New England Journal of Medicine, Vol. 362, No. 17, 2010, pp. 1563-1574. http://dx.doi.org/10.1056/NEJMoa1001282

[4] M. Jun, C. Foote, J. Lv, et al., "Effects of Fibrates on Cardiovascular Outcomes: A Systematic Review and Meta-Analysis,” Lancet, Vol. 375 No. 9729, 2010, pp. 1875-1884. http://dx.doi.org/10.1016/S0140-6736(10)60656-3

[5] S. Kasai, T. Inoue, H. Yoshitomi, et al., "Antidiabetic and Hypolipidemic Effects of a Novel Dual Peroxisome Proliferator-Activated Receptor (PPAR) $\alpha / \gamma$ Agonist, E3030, in $\mathrm{db} / \mathrm{db}$ Mice and Beagle Dogs," Journal of Pharmacological Sciences, Vol. 108, No. 1, 2008, pp. 40-48. http://dx.doi.org/10.1254/jphs.FP0072346

[6] Food and Drug Administration, "Guidance for Industry and Reviewers: Estimating the Safe Starting Dose in Clinical Trials for Therapeutics in Adult Healthy Volunteers,” Draft Guidance, USDHHS, FDA, CDER, CBER, 2002.

[7] T. Hashimoto, H. Kasai, M. Yamada, et al., "Statistical
Assessment of Linear Pharmacokinetics in Clinical Pharmacological Studies,” Xenobiotic Metabolism and Disposition, Vol. 16, No. 3, 2001, pp. 244-252.

[8] Food and Drug Administration, "Guidance for Industry: Food-Effect Bioavailability and Fed Bioequivalence Studies,” FDA, CDER, 2002.

[9] T. M. O’Moore-Sullivan and J. B. Prins, "Thiazolidinediones and Type 2 Diabetes: New Drugs for an Old Disease,” Medical Journal of Australia, Vol. 176, No. 8, 2002, pp. 381-386.

[10] R. R. Henry, A. M. Lincoff, S. Mudaliar, et al., "Effect of the Dual Peroxisome Proliferator-Activated Receptor- $\alpha / \gamma$ Agonist Aleglitazar on Risk of Cardiovascular Disease in Patients with Type 2 Diabetes (SYNCHRONY): A Phase II, Randomised, Dose-Ranging Study,” Lancet, Vol. 374, No. 9684, 2009, pp. 126-135. http://dx.doi.org/10.1016/S0140-6736(09)60870-9

[11] W. C. Roberts, "Safety of Fenofibrate-US and Worldwide Experience,” Cardiology, Vol. 76, No. 3, 1989, pp. 169-179. http://dx.doi.org/10.1159/000174488

[12] G. F. Blane, "Comparative Toxicity and Safety Profile of Fenofibrate and Other Fibric Acid Derivatives," American Journal of Medicine, Vol. 83, No. Suppl. 5B, 1987, pp. 26-36. http://dx.doi.org/10.1016/0002-9343(87)90868-0

[13] G. F. Blane, "Review of European Clinical Experience with Fenofibrate,” Cardiology, Vol. 76, No. Suppl. 1, 1989, pp. 1-13. http://dx.doi.org/10.1159/000174541

[14] B. Davies and T. Morris, "Physiological Parameters in Laboratory Animals and Humans,” Pharmaceutical Research, Vol. 10, No. 7, 1993, pp. 1093-1095. http://dx.doi.org/10.1023/A:1018943613122

\section{Abbreviations}

ADR, adverse drug reaction;

AST, aspartate aminotransferase;

AUC, area under the concentration-time curve from 0 time extrapolated to infinite time;

BMI, body mass index;

$\mathrm{CL} / \mathrm{F}$, apparent clearance;

$\mathrm{C}_{\max }$, maximum observed concentration;

CPK, creatine phosphokinase;

ECG, electrocardiography;

FDA, Food and Drug Administration;

HDL, high-density lipoprotein;
HED, human equivalent dose;

IS, internal standard;

LC/MS/MS, liquid chromatographic-tandem mass spectrometry;

LLOQ, lower limit of quantification;

NOAEL, no-observed-adverse-effect level;

OTC, over-the-counter;

PPAR, peroxisome proliferator-activated receptor;

$\mathrm{QC}$, quality control;

$t_{1 / 2}$, terminal elimination phase half-life;

$\mathrm{t}_{\max }$, time to $\mathrm{C}_{\max }$;

$\mathrm{V}_{\mathrm{z}} / \mathrm{F}$, apparent volume of distribution at terminal phase. 